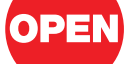

SUBJECT AREAS:

BIOMEDICAL

ENGINEERING

ENDOMICROSCOPY

OPTICAL IMAGING

BRAIN IMAGING

Received

19 December 2012

Accepted

9 April 2013

Published

28 May 2013

Correspondence and requests for materials should be addressed to

A.S. (Asaf.Sh11@ gmail.com)

\section{In vivo minimally invasive interstitial multi-functional microendoscopy}

\author{
Asaf Shahmoon ${ }^{1,2}$, Shiran Aharon ${ }^{3}$, Oded Kruchik ${ }^{3}$, Martin Hohmann ${ }^{1,4,5}$, Hamutal Slovin ${ }^{3}$, \\ Alexandre Douplik ${ }^{1,2,4,5}$ \& Zeev Zalevsky ${ }^{1,2,6}$
}

${ }^{1}$ Clinical Photonics Lab, School in Advanced Optical Technologies (SAOT), Erlangen 91052, Germany, ${ }^{2}$ Friedrich-Alexander Erlangen-Nuremberg University, Erlangen, 91052, Germany, ${ }^{3}$ Multidisciplinary Brain Research Center, Bar-llan University, Ramat-Gan 52900, Israel, " Department of Physics, Ryerson University, 350 Victoria Street, Toronto M5B 2K3, Canada, ${ }^{5}$ Medical Photonics Engineering Group (MPEG), Chair of Photonics Technologies, Erlangen 91052, Germany, ${ }^{6}$ Faculty of Engineering, Bar-llan University, Ramat Gan 52900, Israel.

Developing minimally invasive methodologies for imaging of internal organs is an emerging field in the biomedical examination research. This paper introduces a new multi-functional microendoscope device capable of imaging of internal organs with a minimal invasive intervention. In addition, the developed microendoscope can also be employed as a monitoring device for measuring local hemoglobin concentration in blood stream when administrated into a blood artery. The microendoscope device has a total external diameter of only $200 \mu \mathrm{m}$ and can provide high imaging resolution capability of more than 5,000 pixels. The device can detect features with a spatial resolution of less than $1 \mu \mathrm{m}$. The microendoscope has been tested both in-vitro as well as in-vivo in rats presenting a promising and powerful tool as a high resolution and minimally invasive imaging facility suitable for previously unreachable clinical modalities.

$\mathrm{V}$ arious medical imaging systems, providing information from the depth of bioobjects, can be grouped into invasive and non-invasive. Different types of non-invasive techniques are commercially available, including magnetic resonance imaging (MRI), computed tomography (CT), high and low frequency ultrasound (US), optical coherence tomography (OCT), positron emission tomography (PET), optoacoustic tomography $(\mathrm{OAT})^{1}$ and multispectral optoacoustic tomography (MSOT) ${ }^{2}$. Since each one of these technologies has its own advantages and disadvantages, it motivated combining two or more complementary technologies into a multimodal method exploiting advantages of each - such as PET-MRI ${ }^{3}$, PET-CT ${ }^{4,5}$, FMT-CT $^{6}$ or FMT-MRI ${ }^{7}$. These imaging techniques provide visual sectioning, but they are limited in terms of spatial resolution, whereas OCT provides the best resolution which is about a few micrometers. There is also a clinical demand to estimate some functional biological parameters such as, for instance, local hemoglobin concentration $(\mathrm{Hb})$ for tracking brain hemodynamics affected by external sensory stimulations ${ }^{8,9}$ and monitoring tumor treatment progression ${ }^{10}$. The local hemoglobin fraction can be estimated using PET, MRI, near-infrared spectroscopy (NIRS) and photoacoustic microscopy $(\mathrm{PAM})^{11-13}$. The non-invasive techniques also suffer from limited penetration depth results with the inability to access deep internal organs as well as lack to perform long time observation ${ }^{14}$, whereas minimally invasive micro-endoscopes is a requisite strategy enables the insertion of the microendoscope device directly to the inspected area and enable the implantation of the device to perform long time observation where tracking of disease progression and for on-line evaluation of different therapies are required.

Minimally invasive multi core based fiber bundle as well as rigid microendoscopes have been already demonstrated as an accurate and powerful tool when a higher spatial resolution imaging is required.

The traditional way of microendoscope production is using a high resolution gradient refractive index $(\mathrm{GRIN})^{14-19}$ microlenses, for instance for in-vivo one ${ }^{16}$ and $\mathrm{two}^{16,17,20}$ photon fluorescence, multi-photon fluorescence $^{21,22}$, second harmonic generation (SHG) and confocal fluorescence microendoscopic techniques. Until now, however, these techniques have been limited by the mechanical rigidity, length and endoscopes diameter that limits the accessibility to reach deep internal organs without damaging and affecting their functionality. Minimizing the device diameter is especially critical while performing neurological research ${ }^{23}$.

Another imaging configuration is based on fiber bundles. In this case, the imaging device consists of a few thousand and up to a few tens of thousands of step-index single mode fibers which are incorporate together to perform the required image. Several fiber bundles are commercially available (e.g., Mauna-Kea, Sumitomo and Fujikura) and are being in used for applications as confocal microscopy ${ }^{24,25}$ and two photon imaging ${ }^{26}$. 
To overcome the limitations of GRIN endoscopes and to improve the performance of the currently available fiber bundles, we introduce a special polymer micro endoscopic system having a total diameter of less than $200 \mu \mathrm{m}$ (Fig. 1). The system can penetrate tissues in minimally invasive manner or go through blood vessels to reach the required inspected area. The field of view (FOV) obtained from zero distance and the lateral resolution of the microendoscope are $200 \mu \mathrm{m} \times 200 \mu \mathrm{m}$ and $900 \mathrm{~nm}$, respectively.

Despite the thin diameter of the microendoscope it can generate an image with relatively large field of view (FOV) using post processing algorithm by stitching sequentially captured images and with a millisecond scale resolution. The design of the microendoscope takes into account the size of the core and the difference between the refractive indexes of the cladding and the core of each of the 5,000 optical cores (pixels) and supports only single mode operation ${ }^{27}$ whereas multi mode signals ${ }^{27}$ cannot develop along the microendoscope device and thus the constructed image is therefore insensitive to fiber's bending. This important property, allows the navigation of the microendoscope system during the medical imaging procedure along curved veins without distorting the image constructed. In contrast to GRIN based microendoscopes, which are rigid and short, the suggested device enables mechanical flexibility at the same time maintaining a certain stiffness as fabrication of more than one meter lengths scopes is applicable. Hence, it can be implanted in a living subject to perform even chronic studies over a longer time interval ${ }^{14}$ (longitudinal system). The optical microendoscope is composed from two kinds of polymers. The core is made from polysterene (PS) and the cladding is fulfilled from poly(methyl methacrylate) PMMA.

In respect to its imaging related property, the microendoscope is actually a transmission medium transferring the optical wavefront arriving from the object to its input edge through the optical cores of the scope towards its outlet from which it continues to propagate towards the resulted image plane. The obtained imaging relation is:

$$
\frac{1}{U_{1}+U_{2}}+\frac{1}{V}=\frac{1}{F}
$$

where $U_{1}, U_{2}$ and $V$ are the distances between the object and the end of the micro endoscope, the end of the micro endoscope and the imaging lens and the imaging lens and the image detection array (CCD plate), respectively (Fig. 1). $F$ is the focal length of the imaging lens. The suggested system does not involve a lens or an array of micro-size lenses at the end of the imaging tip and thus the entire imaging concept is novel and different from what is common nowadays in the field of endoscopy. The array of cores allows to sense and backwards transmit the wave front of the back reflected light and the imaging capability resembles what is done in Shack-Hartmann interferometers in ophthalmology. Due to that the natural working distance is close to be in contact with the examined area. However, since we do not use a lens configuration at the end of the microendoscope tip, the classical relations/trade-offs between resolution, field of view and depth of focus are not fully reserved in our case. Thus, the working distance can be adapted to a different value (up to $0.5 \mathrm{~mm}$ ) by changing the relative distance of the external imaging lens located between the CCD camera and the external end of the microendoscope tip.

The performance of the system (Fig. 1) and the results acquired from in-vivo imaging of living rats are presented in the Results section below. In addition, in order to illustrate the ability of the microendoscope to be used also as a monitoring device, we have measured localized hemoglobin concentration inside a phantom. The phantom mimics human skin in terms of optical properties such as absorption, scattering coefficients and phase function of scattering (anisotropy factor).

The microendoscope device has been used for imaging of fluorescence protein in which HEK 293 cells were transfected with pEGFP-N3. $48 \mathrm{hrs}$ later the cells were observed (Supplementary Fig. 1; Supplementary method). Additional resolution targets have been imaged using the microendoscope to emphasis the performance

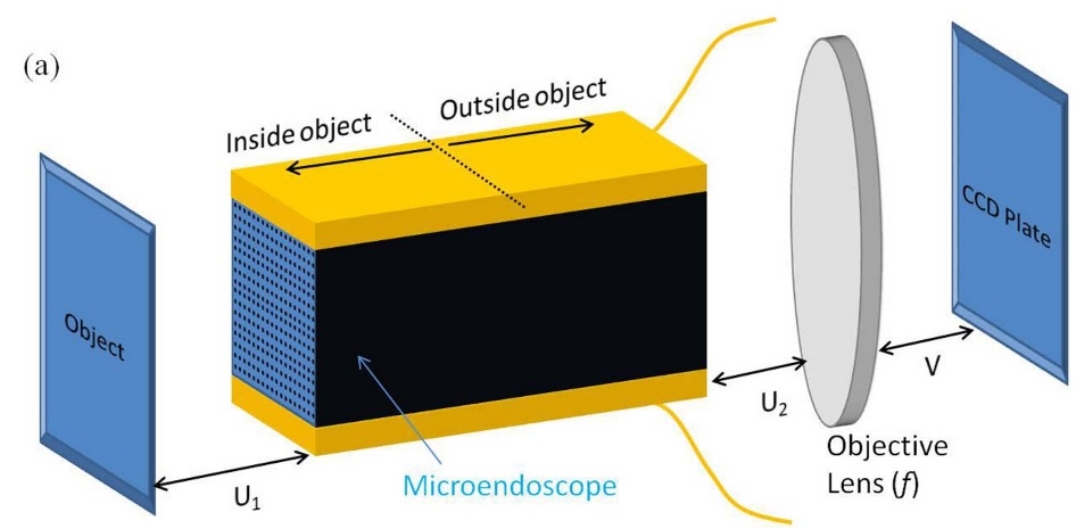

(b)
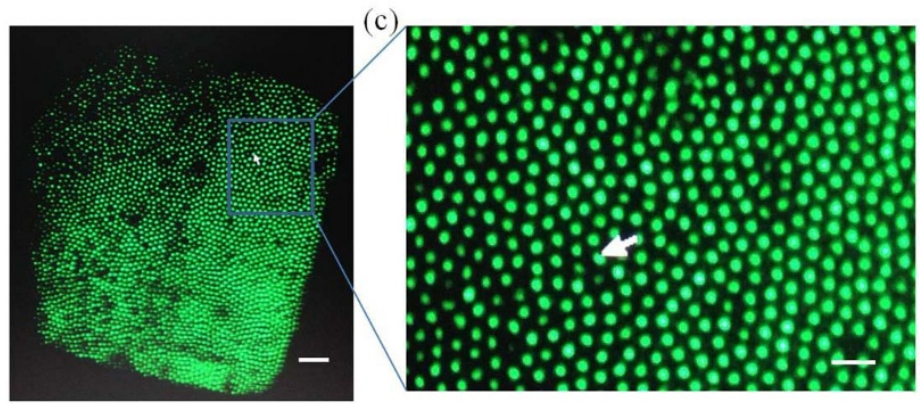

Figure $1 \mid$ Schematic description of the imaging operation principle of the microendoscope. (a) experimental setup for in-vivo and in-vitro applications used for monitoring and imaging. (b) top view microscope image of the cross section of the microendoscope. (c) zooming of the margined area in b. Scale bars in b and c are 20 and $5 \mu \mathrm{m}$, respectively. 
and real time acquisition (Supplementary Fig. 2 and Supplementary Video 1).

\section{Results}

Imaging of rat brain microvascular. We present in-vivo studies obtained by using the microendoscope system (Fig. 1). The studies were performed on anaesthetized rats. The microendoscope was inserted into the rat's brain through the barrel cortex. The imaging of the microvascular area was performed without removing the dura layer but rather imaging it from above (Fig. 2a). During the experiment we used green light laser at wavelength of $532 \mathrm{~nm}$ (Fig. 2b-e) in order to highlight the vein's contrast of the micro vascular area inside the rat brain. Different veins widths (Fig. 2b,c) as well as different veins brunches converged ultimately into a single larger vein (Fig. 2d-e). The contours of the veins are marked by the solid red lines. Smaller venules captured within the microendoscope field of view, are indicated by the solid arrows (Fig. 2d-f). In addition, we used red light laser at wavelength of $642 \mathrm{~nm}$ to image two vein brunches combined into a single larger vein located at a lower interstitial layer. The image was acquired using a low signal camera. This demonstrates that the microendoscope can successfully deliver low-level signals from deep internal layers. The navigation of the microendoscope inside the brain tissue has been done by a five axis positioning stage (XYZ plus tilt and rotation platform). On top of it, we positioned a V-groove bare fiber holder, which allows locating and navigating the microendoscope across the area of interest.

Imaging of blood veins of a chicken wing. We further explored the microendoscope imaging system $(200 \mu \mathrm{m}$ total diameter) by examining its ability to reproduce an image with an extended FOV, although the total diameter of the microendoscope is only $200 \mu \mathrm{m}$. To illustrate it, we used the microendoscope in order to continuously image a blood vein of a chicken wing (Fig. 3a-d). Each of the presented images shows different location of the microendoscope along the vein. The images are individually shown instead of stitched into a single image illustrating an extended FOV of the complete image along the blood vein. The solid arrows indicate the observed vein, while the dashed arrows as well as the labeling letter indicate the stitching point between the images (Fig. 3a-d). The extended FOV image is constructed by calculating the relative movement of the multi axis platform at which the microendoscope is located, and converting it into the movement of the image generating ultimately a larger image in a real time processing. If such a stitching procedure has been conducted properly, then a full length of the examined area of interest (e.g. blood vein) can be reproduced as a single image.

To further illustrate the ability of the microendoscope system to be inserted into an internal organ and to generate high resolution images, we imaged a tiny blood vein from a chicken wing tissue. The encompass area of the top view microscope image (Fig. 3e) has been imaged using the microendoscope system showing imaging along a $4 \mu \mathrm{m}$ (Fig. $3 \mathrm{f}-\mathbf{h})$ blood vein at different locations.

Imaging inside a phantom. A key advantage of our microendoscope system over existing microendoscopes concerns the very thin external diameter which allows penetration inside an examined area with minimal invasive damage or going along proper vascular vein directly to the examined area. Nonetheless, although the thin diameter, of the microendoscope, it can provide images at high spatial resolution of about 5,000 pixels and a FOV of $200 \mu \mathrm{m}$ (when operating in contact mode).

We further explored the capabilities of the microendoscope system to be inserted inside the object of study. The microendoscope was slided through a channel drilled inside a light scattering phantom (Fig. 4a) mimicking human skin. We illustrate the applicability of the microendoscope to be inserted inside a small blood vessel in order to reveal partial or full clogging of the vessel. For this
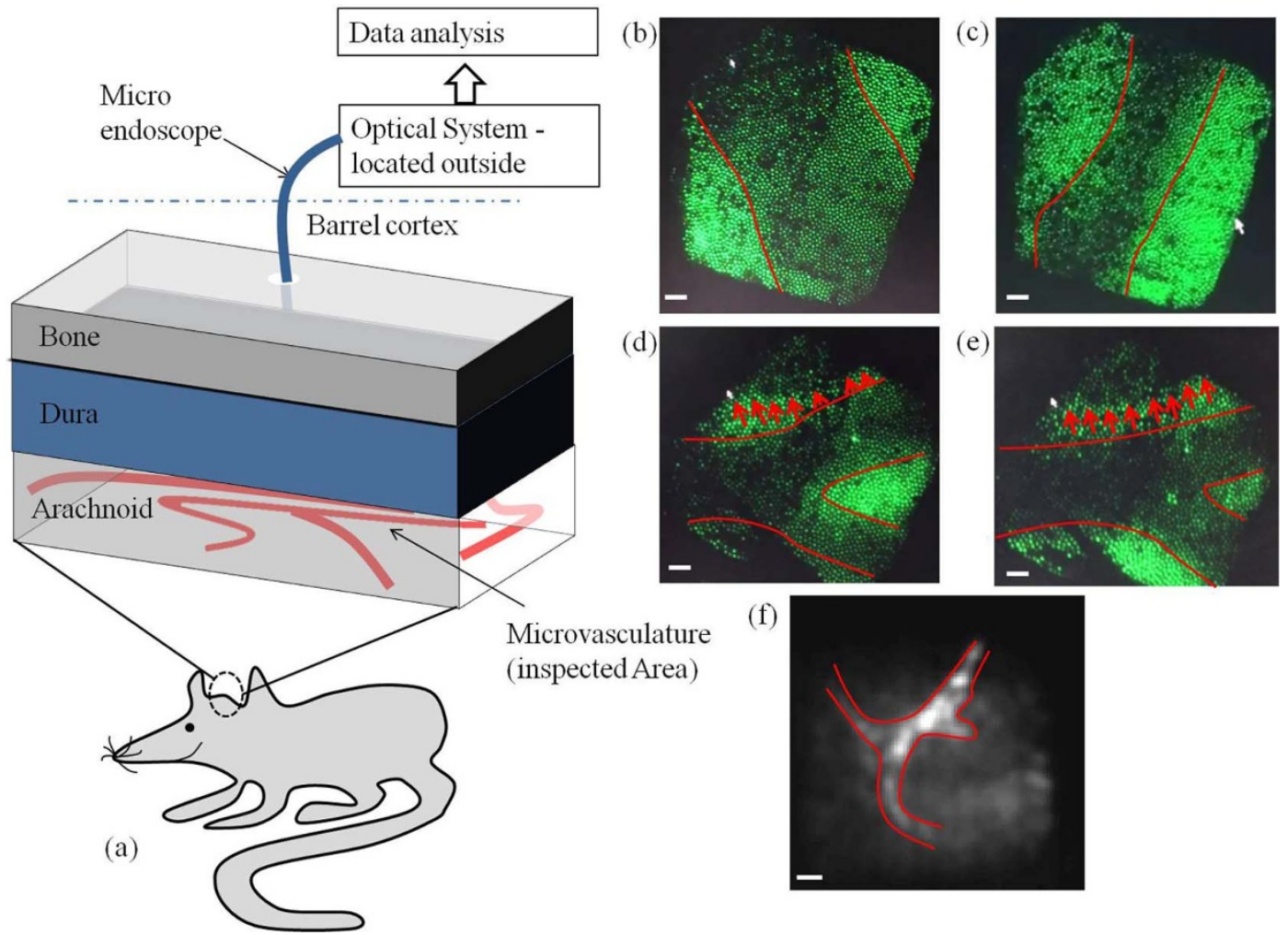

Figure $2 \mid$ Microendoscopic in-vivo imaging experimental setup. (a) Schematic sketch of the rat and the insertion through the barrel cortex of the rat's brain. (b-c) different size of blood veins indicated by the solid red lines. ( $d-f$ ) vein brunches converging into a larger vein. (f) low signal image captured using MiCam ULTIMA camera. Scale bars are of $20 \mu \mathrm{m}$. 

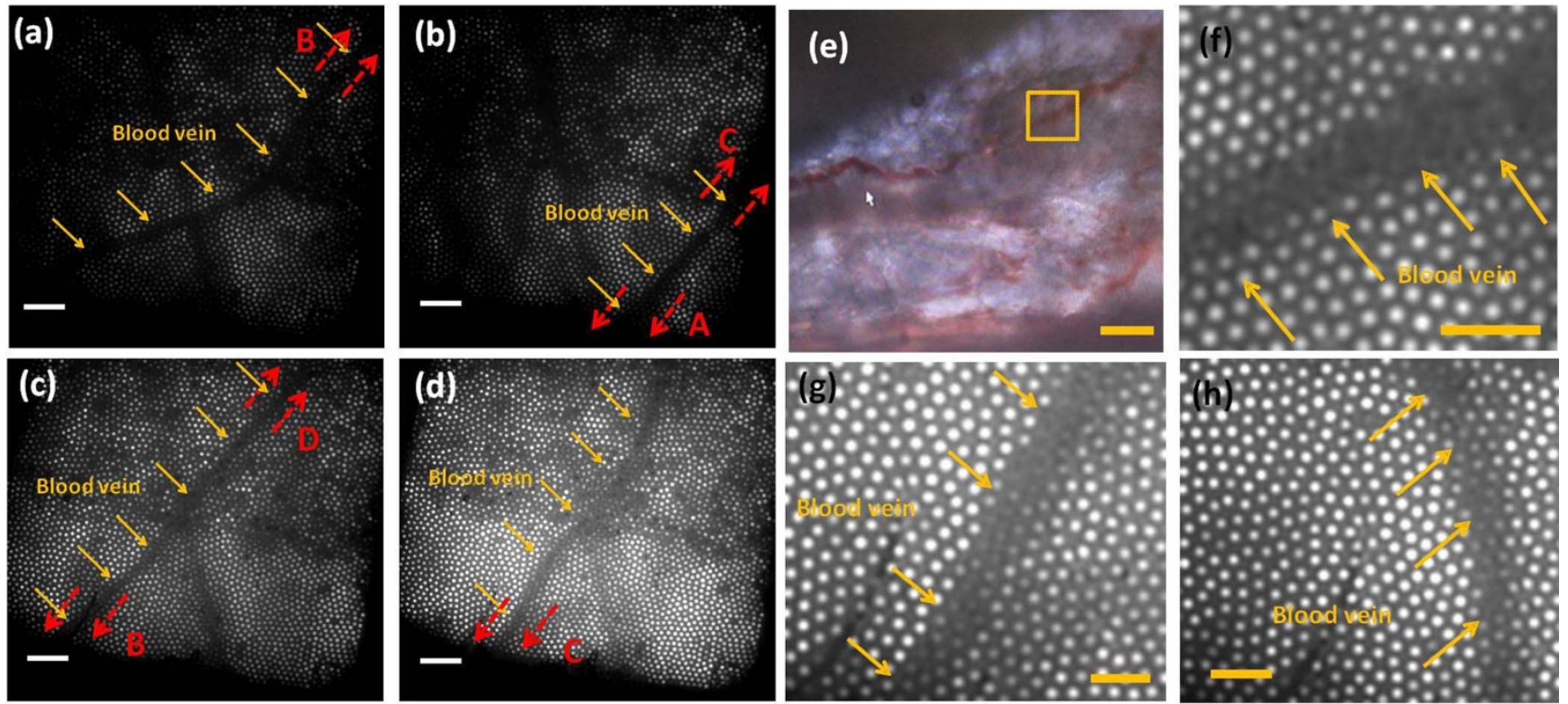

Figure 3 Blood veins images from a chicken tissue. (a)-(d) Imaging along a blood vein of a chicken wing sliding over the surface. The solid arrows indicate the blood vein, while the dashed arrows and labeling letter indicate the "stitching" point between the images for generating an image with an extended field of view. (e) Top view microscope image of a tiny blood vein. (f)-(h) Imaging at different locations along a $4 \mu \mathrm{m}$ blood vein (Shown in e) inside a chicken wing. Scales bar in (a)-(e) and (f)-(h) are 20 and $4 \mu \mathrm{m}$, respectively.

experiment we used the same microendoscope prototype as above. The 3D-sketch of the phantom (Fig. 4b) shows two channels drilled with diameter of $400 \mu \mathrm{m}$ each. One longitudinal channel (along the $\mathrm{x}$ axis) was drilled all the way through the phantom block (Fig. 4c), while another angled channel (Fig. 4d) was made making both channels cross linked inside the phantom. The openings indicated as "in" and "out" formed a flow through system connected with a syringe (Desoi GmbH, Gemany) at the entrance ("in"). The crosssectional schematic view (Fig. 4c,d) of the fabricated phantom shows the paths of the channels. Syringe needles (19G) were inserted into
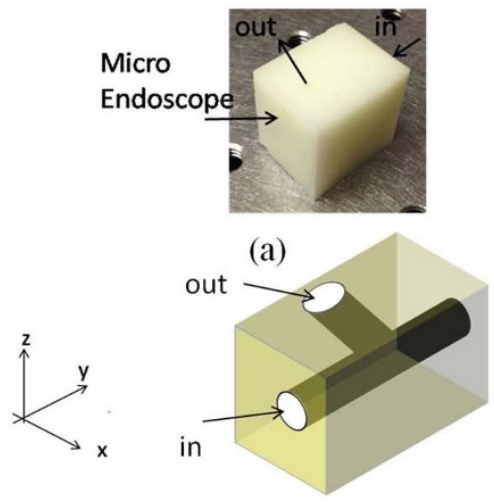

(b)

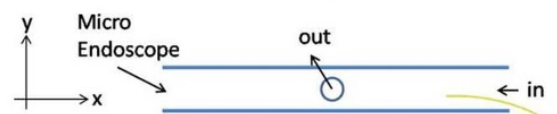

(c)

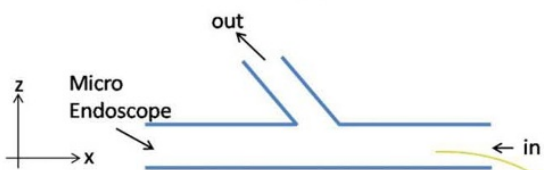

(d)
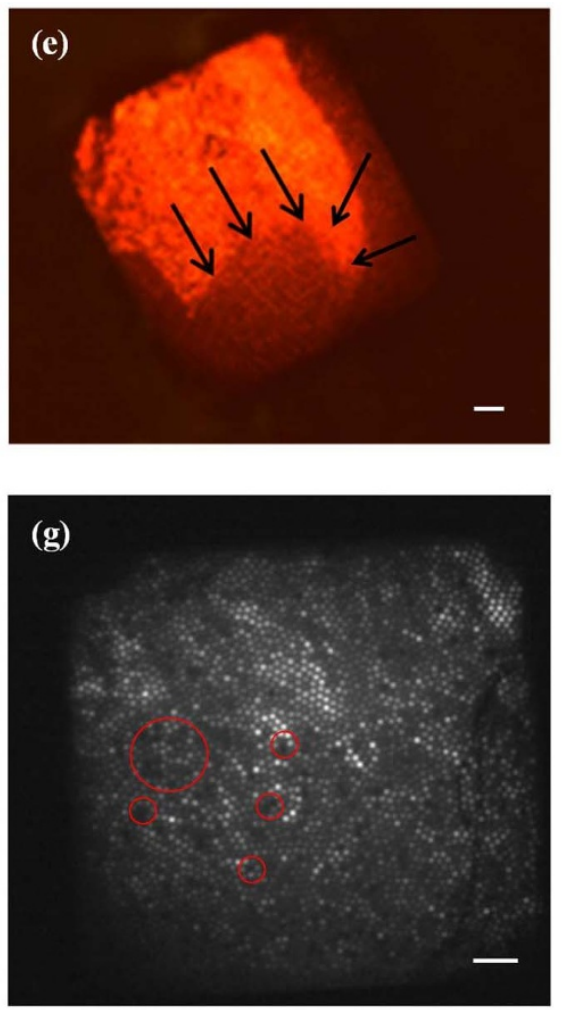

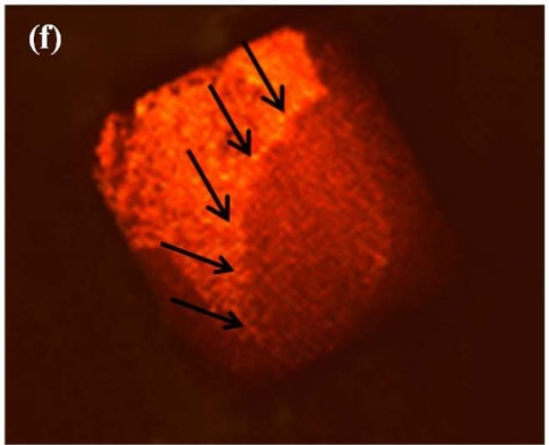

(h)

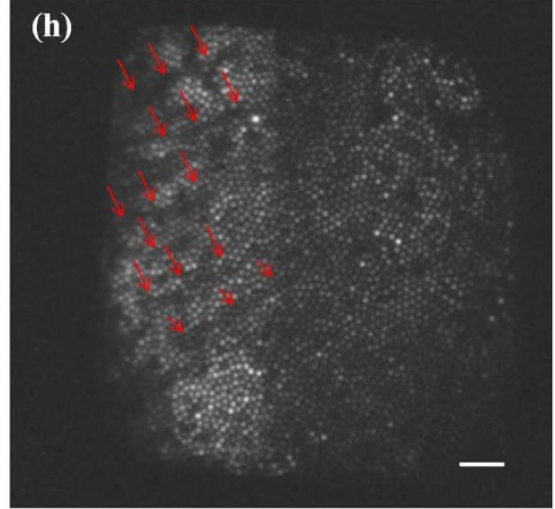

Figure 4 Experimental studies inside a phantom (a) fabricated phantom. (b) 3D view sketch of the phantom having two drilled channels at diameter of $400 \mu \mathrm{m} .(\mathrm{c}, \mathrm{d})$ cross-sectional views of the channels inside the phantom. (e,f) Imaging of a manipulated micro wire (indicated by the solid arrows) inside an hemoglobin mixture. (e) and (f) shows the imaged micro wire at different orientation and position. (g,h) imaging of magnetic micro particles inside the intralipid mixture. (g) Spares distribution of the micro particles along the micro probe interface. (h) Imaging of the magnetic micro particles under external magnetic field. 
the both openings in the phantom to deliver and exhaust fluid. A micro wire (stainless steel, 150 micro) imitating a partial blocking of a blood vessel, was inserted from the "in" opening. A mixture of $1 \%$ intralipid (IL) and 5\% human hemoglobin (Aldrich Sigma, Germany) and $94 \%$ water (all three components mixed up were imitating human blood) was used as a solution flowing through the phantom channels. The other opening ("out") was employed to slide the microendoscope inside the phantom to make it face the micro wire. Different orientation as well as positions of the manipulated micro wire (Fig. 4e,f) inside the channel are marked in the figure by several solid arrows. These images clearly validate the circular shape of the micro wire.

In order to further demonstrate the high spatial discrimination of the microendoscope and its ability to image micro size features, we used a mixture of $2 \%$ intralipid (IL), $2 \%$ magnetic micro particles (MMPs) and $96 \%$ water as a solution flowing through the channels inside the phantom. The MMPs had diameter of $3 \mu \mathrm{m}$.

The MMPs inside the intralipid mixture demonstrates a random distribution (Fig. 4g) along the microendoscope interface. As it can be seen, several micro particles are encompassed inside the red circles depicted on the image. After applying external magnetic field which was performed using an external magnet (Super-strong Rare-Earth RE Magnets, external diameter of $8 \mathrm{~mm}$ ), the MMPs have been aggregated into an elongated shape according to the position and the orientation of the external magnetic field (Fig. $4 \mathrm{~h}$ and Supplementary Video 2). The MMPs aggregations are marked by the solid red arrows.

(a)

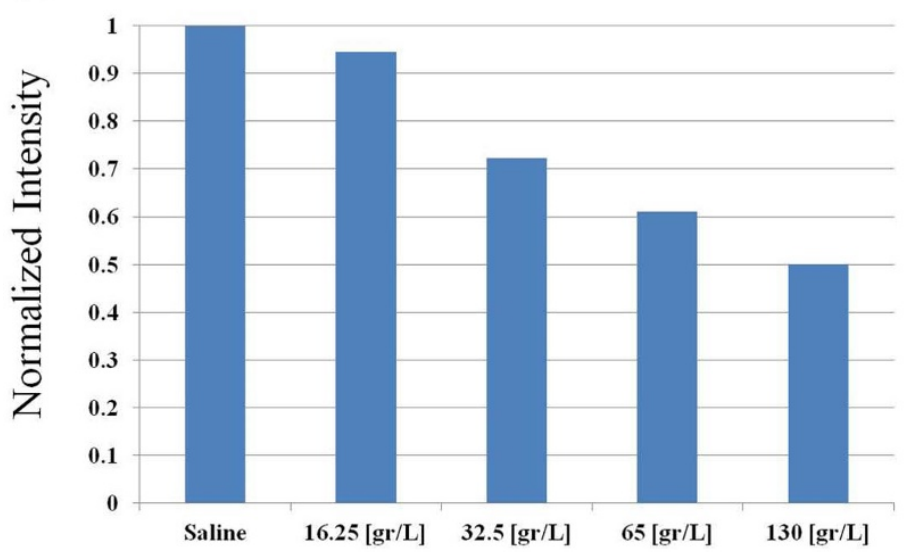

\section{Concentration level}

(c)

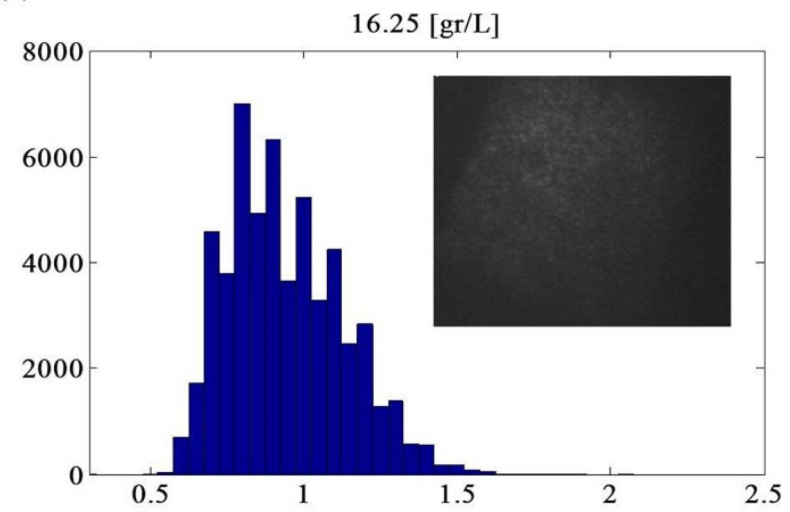

Monitoring of localized hemoglobin concentration. In order to further demonstrate the applicability and the multi functionality of the proposed microendoscope system, to be used not only as a minimally invasive imaging device but also as a biological monitoring device, we have measured localized oxygenated hemoglobin concentrations. To illustrate it, we injected and circulated different hemoglobin concentrations through the aforementioned phantom (Fig. 4a,b) while inserting the microendoscope device through the phantom's drilled channels. Different oxygenated human hemoglobin concentration levels have been tested. We used the saline solution as a control level for normalizing the other measurements of the hemoglobin concentrations (Fig. 5a). A laser illumination source at wavelength of $532 \mathrm{~nm}$ was used to illuminate the hemoglobin solutions, whereas the difference between the intensities absorbance reflects the difference between the hemoglobin concentrations. Normalized histograms (Fig. 5b-d) reflecting the distribution of the insets images of saline and hemoglobin concentrations of 16.25 [gr/L] and 130 [gr/L], respectively. The histograms were normalized by dividing the maximum level imaged by the saline solution to allow visual comparison with respect to the control level of the saline.

\section{Discussion}

We have introduced a new multi-functional micro endoscopic system having micro sized diameter being very applicable for minimally invasive in-vivo imaging as well as for monitoring of localized

(b)

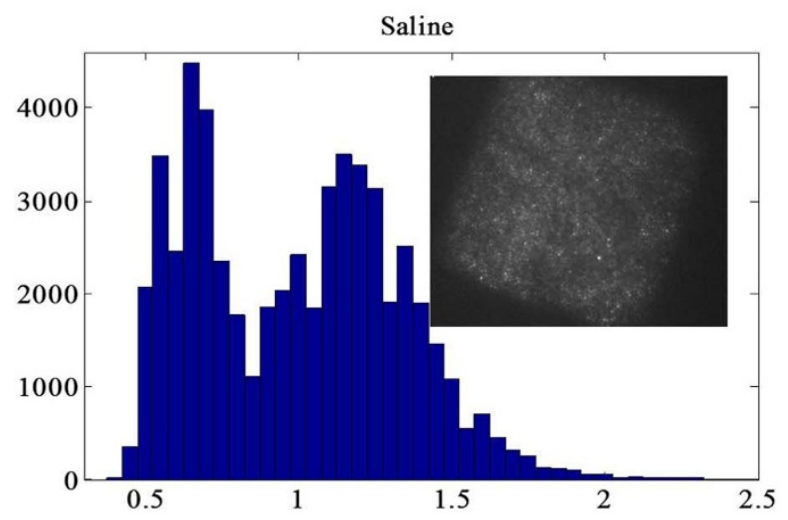

(d)

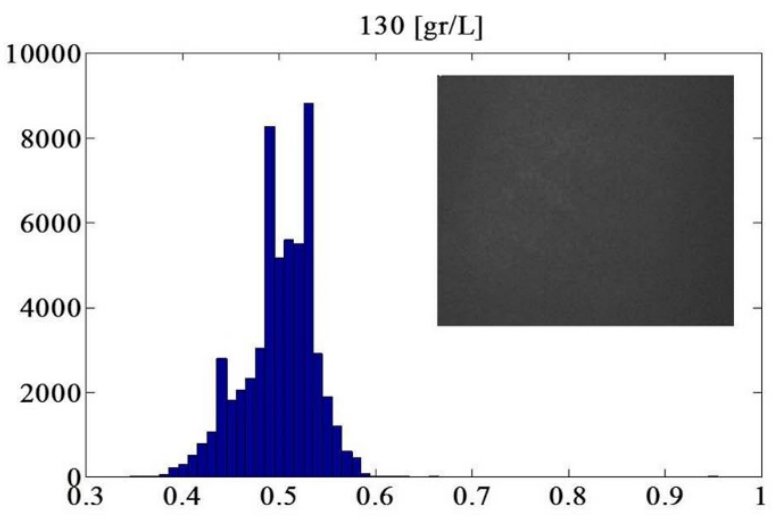

Figure 5 Monitoring hemoglobin concentration inside a phantom. (a) Normalized intensities for characterization of different hemoglobin concentrations. (b)-(d) Normalized histograms reflecting the distribution of the insets images of saline and hemoglobin concentrations of 16.25 [gr/L] and $130[\mathrm{gr} / \mathrm{L}]$, respectively. 
hemoglobin concentration. The micro size diameter of the proposed endoscopy system allows medical imaging from deep internal organs in minimally invasive way. Moreover, due to its thin diameter, the microendoscope device can access the body by venipuncture and by penetrating it along a blood vein acting as a channel guiding the device towards the inspected area. The combination of the micro size diameter and the multi-functionality capabilities offers a new method for facilitating localized bio medical imaging as well as biological monitoring. In this paper we focused on imaging of microvasculature. However, the proposed micro endoscopy approach can be extended to other areas as illustrated in the phantom experiments (Fig. 4).

A key advantage of the proposed micro endoscopy system is related to the fact that it offers a disposable device due to the low cost of its manufacturing process. Whereas, the currently existing microendoscopes are mainly being in use for multiple patients which obviously increases the risk for cross-contamination. In addition, the micro sized diameter, flexibility and the length of the device enable real time observations (longitudinal studies) by implanting the device into the area of the examined subject while having full freedom of movement without causing any feeling of inconvenience. Longitudinal studies may provide deep knowledge over a real time process of an in-vivo diseases progression ${ }^{20}$ and correlating between the information gathered along the period of observation. This leads to a more accurate understanding over the early stage of the symptoms, the development of the disease and the therapies that should be taken.

Although the microendoscope device may pave the way for new medical examinations, it still has some limitations that should be mentioned. First, the device is being constructed from two types of polymers which form the optical core and the cladding of the propagating signal. Those polymers are mainly suitable for the visible spectrum, especially for wavelengths at range of $500-700 \mathrm{~nm}$. Beyond this spectral range, the propagating signal is being absorbed by the polymers. Second, the polishing phase of the device is not followed by a regular splicing process as the one existing with regular silica made fibers. It is rather a manual polish that is carried out using a sensitive procedure involving, among other several types of sand papers.

In the experimental results we were able to obtain imaging from inside of a microvasculature area inside rat's brain. In addition, imaging of a micro wire as well as MMPs inside different mixtures inside channels drilled through phantoms were presented. The micro wire is imitating a partial block of a blood vessel, while the MMPs demonstrate the ability to distinguish between micro size features.

The demonstrated capabilities showed in the experimental results were done with a microendoscope prototype having external diameter of $200 \mu \mathrm{m}$ which produces an image of about 5,000 pixels before applying super resolving techniques. After elaborating super resolution ${ }^{28,29}$ the obtainable resolution limit as well as the field of view will exceed the state of the art resolution and field of view of nowadays available in conventional microendoscopes. The presented experimental results were performed by applying the fabricated optical hardware and without using any super resolving approaches.

Another key consideration that should be taken into account when designing such an advanced micro endoscopy device is the capability to generate a one-piece tool that enables not only imaging of internal organs but also monitoring of biological parameters as well as treating them using the same endoscope. The microendoscope device basically back transmits the wavefront reflected from the inspected object and generates the image. However the optical cores can also be used to illuminate the object itself or even to heat it if illuminated with high photonic power density. In addition, the rectangular shape of the microendoscope device enables sputtering of two electrically conductive layers made out of different metallic materials (Fig. 1a) on top of its two faces in order to be able to generate electrical heating at the tip of the endoscope. This feature can be very useful for different applications such as blocking of blood vessels e.g. against "cold womb" or for treating prostate cancer or for thermotherapy.

Overall, the proposed multi-functional micro endoscopy system can potentially enable studies from deep and sensitive internal organs such as understanding brain disorders and diseases in the nervous system ${ }^{23,30}$ and to reveal how damages in the nervous system affect the behavior of a single cell by simultaneously correlating the level of the localized hemoglobin concentration to brain functionality ${ }^{31}$.

In respect to the general characterization of the working envelop of the proposed microendoscope, the FOV for close to zero distance is $200 \mathrm{um} \times 200 \mathrm{um}$ and the lateral resolution is $900 \mathrm{~nm}$. The WOD can be changed from zero and up to a few millimeters. Moving the tip of the microendoscope backwards from the object will result with an increased FOV. The microendoscope can be bend up to 270 deg with a diameter of less than $8 \mathrm{~mm}$ (depends on the articulation system). Super resolution techniques can improve the resolution by at least an order of magnitude. The above mentioned parameters can be modified and adapted according to the specific designated application and the required resolution, FOV, WOD and working channel. It is also important to note that the tradeoffs between FOV, resolution, WOD are not restrict as in regular imaging configurations as we do not use an imaging lens at the end of the microendoscope but rather transmit the wavefront of the back reflect light (similar to Shack-Hartmann interferometers).

\section{Methods}

Microendoscope device. The microendoscope device is made out of polymers, while the core is made out of PS (Polystyrene) and the cladding is made out of Poly(methyl methacrylate) (PMMA). The refractive indexes of the PS and the PMMA at a wavelength of $642 \mathrm{~nm}$ are 1.59 and 1.49, respectively. For $532 \mathrm{~nm}$ the refractive indexes of the PS and PMMA are 1.597 and 1.495, respectively. We have fabricated multi core fibers having very thin external diameters of $100 \mu \mathrm{m}$ and $200 \mu \mathrm{m}$ that both of them have the same number of pixels (i.e. different core to cladding ratio). For the $200 \mu \mathrm{m}$ prototype the number of optical cores is about 5,000, whereas the FOV at zero distance is $200 \mu \mathrm{m} \times 200 \mu \mathrm{m}$. The lateral resolution of the microendoscope is $900 \mathrm{~nm}$.

Rat preparation. Surgical procedure of the rat involved previously described works $^{32,33,29}$. In brief, the rat has been placed in a cage whereas isofleurane is injected through a vaporizior. Right after, the rat is anaesthetized using injection of urethane and positioned at a special rat head holder. Then an approximately $7 \mathrm{~mm}$ diameter hole is drilled above the barrel cortex of the rat, which is identified by the anatomical coordinates, while the imaging of the rat brain microvascular have been performed from above the dura layer. All rats experiments were approved by the Animal Research Facility at Bar-Ilan University.

Phantom. The phantom was made out of two component polyurethane WC 781 A/B. $100 \mathrm{ml}$ of component A and $100 \mathrm{ml}$ of component B were used for processing. Inside component A we added $1.4 \mathrm{~g}$ titanium oxide. Both mixtures were properly mixed separately using a spoon. Afterwards, there were homogenized inside an ultrasonic bath (Elmasonic P $30 \mathrm{~h}$, Elma $\mathrm{GmbH}$ ). The ultrasonic modes were adjusted to degas, sweep and pulse without heating. Right after, each mixture was degased inside a degasser with a pressure of approximately $0.0001 \mathrm{bar}$. This aforementioned process was repeated one time for getting a homogeneous mixture without gas bubbles. Each step of the degassing and the ultrasound was done for at least 5 and $15 \mathrm{~min}$, respectively. Afterwards the components were carefully mixed. The $400 \mu \mathrm{m}$ channels along the phantom were drilled using an accurate mechanical micro driller.

Optical instrumentation. To capture the light signals coming from the object and propagates along the microendoscope, we used either a $10 \times, 0.25$ - numerical aperture microscope objective lens (Motic, EFNPlan) to perform the imaging of the chicken wing (Fig. 3) and the result acquired from the phantom (Fig. 4) or a $20 \times$, 0.4 - numerical aperture microscope objective lens (Nikon,CEIPlan) to perform the cross sectional view of the microendoscope (Figs. 1b,c) and the rat studies (Fig. 2). Charged coupled device (CCD) camera (DXM-1200, Nikon) has been used to capture the images of Fig. $\mathbf{1}$ and of Fig. $\mathbf{2 b}-\mathbf{e}$, whereas a CMOS imaging system (MiCam ULTIMA, SciMedia) was used to capture Fig. 2h. We used Moticam 2000 (Motic) camera to perform imaging of the chicken wing (Fig. 3), the results acquired from the phantom experiment (Fig. 4) and the images used for the analysis of the hemoglobin concentration (Fig. 5). A Laser illumination at wavelength of $532 \mathrm{~nm}$ (Suwtech, Shahghai Uniwave Technologies) was used as an illumination source for the rat and 
the chicken wing studies as well as for monitoring the localized hemoglobin concentration. Laser illumination at wavelength of $642 \mathrm{~nm}$ (He-Ne gas laser, JDS Uniphase) was used in the phantom experiments (Fig. 4).

The excitation power used during the rat brain microvascular imaging was measured using Nova - Laser Power Meter (Ophir Products, Israel) to a value of about $13 \mathrm{~mW}$.

Analysis software. Calculating the localized hemoglobin concentration has been carried out using a fundamental computational tool of Matlab (MathWorks).

1. Wang, X. et al. Noninvasive laser-induced photoacoustic tomography for structural and functional in vivo imaging of the brain. Nature Biotechnol. 21, 803-806 (2003).

2. Razansky, D. et al. Multispectral opto-acoustic tomography of deep-seated fluorescent proteins in vivo. Nature Photonics 3, 412-417 (2009).

3. Judenhofer, M. S. et al. Simultaneous PET-MRI: a new approach for functional and morphological imaging. Nature Medicine 14, 459-465 (2008).

4. Beyer, T. et al. A combined PET/CT scanner for clinical oncology. The Journal of nuclear medicine 41, 1369-1379 (2000).

5. Ciernik, I. F. et al. Radiation treatment planning with an integrated positron emission and computer tomography (PET/CT): a feasibility study. International Journal of Radiation Oncology, Biology, Physics 57, 853-863 (2003).

6. Grimm, J. et al. Use of gene expression profiling to direct in vivo molecular imaging of lung cancer. PNAS 102 (1), 14404-14409 (2005).

7. Leimgruber, A. et al. Behavior of Endogenous Tumor-Associated Macrophages Assessed In Vivo Using a Functionalized Nanoparticle. Neoplasia 11, 459-468 (2009).

8. Brecht, M. \& Sakmann, B. "Dynamic representation of whisker deflection by synaptic potentials in spiny stellate and pyramidal cells in the barrels and septa of layer 4 rat somatosensory cortex". J. of Physiology 543 (1), 49-70 (2002).

9. Vanzetta, I. \& Grinvald, A. Increased Cortical Oxidative Metabolism Due to Sensory Stimulation: Implications for Functional Brain Imaging. Science 286, 1555-1558 (1999).

10. Menon, C. \& Fraker, D. L. Tumor oxygenation status as a prognostic marker. Cancer Letters 221, 225-235 (2005).

11. Zhang, H. F., Maslov, K., Sivaramakrishnan, M., Stoica, G. \& Wang, L. V. Imaging of hemoglobin oxygen saturation variations in single vessels in vivo using photoacoustic microscopy. Applied Physics Letters 90, 053901-3 (2007).

12. Zhang, H. F., Maslov, K., Stoica, G. \& Wang, L. V. Functional photoacoustic microscopy for high-resolution and noninvasive in vivo imaging. Nature Biotechnology 24, 848-851 (2006).

13. Wang, X., Xie, X., Ku, G., Wang, L. V. \& Stoica, G. Noninvasive imaging of hemoglobin concentration and oxygenation in the rat brain using high-resolution photoacoustic tomography. J. Biomed. Opt 11, 024015-024015 (2006).

14. Flusberg, B. A. et al. Fiber-optic fluorescence imaging. Nat Methods 2, 941-950 (2005)

15. Llewellyn, M. E., Barretto, R. P. J., Delp, S. L. \& Schnitzer, M. J. Minimally invasive high-speed imaging of sarcomere contractile dynamics in mice and humans. Nature 454, 784-788 (2008).

16. Jung, J. C., Mehta, A. D., Aksay, E., Stepnoski, R. \& Schnitzer, M. J. In Vivo Mammalian Brain Imaging Using One- and Two-Photon Fluorescence Microendoscopy. J Neurophysiol 92, 3121-3133 (2004).

17. Flusberg, B. A., Jung, J. C., Cocker, E. D., Anderson, E. P. \& Schnitzer, M. J. In vivo brain imaging using a portable 3.9 gram two-photon fluorescence microendoscope. Opt. Lett. 30, 2272-2274 (2005).

18. Barretto, R. P. J., Messerschmidt, B. \& Schnitzer, M. J. In vivo fluorescence imaging with high-resolution microlenses. Nat Methods 6, 511-512 (2009).

19. Deisseroth, K. et al. Next-Generation Optical Technologies for Illuminating Genetically Targeted Brain Circuits. J Neurosci 26, 10380 (2006)

20. Huland, D. M. et al. In vivo imaging of unstained tissues using long gradient index lens multiphoton endoscopic systems. Biomed. Opt. Express 3, 1077-1085 (2012).
21. Levene, M. J., Dombeck, D. A., Kasischke, K. A., Molloy, R. P. \& Webb, W. W. In vivo multiphoton microscopy of deep brain tissue. J. Neurophysiol. 91, 1908-1912 (2004).

22. Murray, T. A. \& Levene, M. J. Singlet gradient index lens for deep in vivo multiphoton microscopy. J. Biomed. Opt 17, 021106-1 (2012).

23. Misgeld, T. \& Kerschensteiner, M. In vivo imaging of the diseased nervous system. Nature Reviews Neuroscience 7, 449-463 (2006).

24. Carlson, K. et al. In vivo fiber-optic confocal reflectance microscope with an injection-molded plastic miniature objective lens. Appl. Opt. 44, 1792-1797 (2005).

25. Juskattis, R., Wilson, T. \& Watson, T. F. Real-time white light reflection confocal microscopy using a fibre-optic bundle. Scanning 19, 15-19 (1997).

26. Göbel, W., Kerr, J. N. D., Nimmerjahn, A. \& Helmchen, F. Miniaturized twophoton microscope based on a flexible coherent fiber bundle and a gradient-index lens objective. Opt. Lett. 29, 2521-2523 (2004).

27. Saleh, B. E. A. \& Teich, M. C. Fundamentals of photonics (Wiley-Interscience: 2007).

28. Zalevsky, Z. \& Mendlovic, D. Optical Super Resolution. Springer (2004)

29. Shemer, A., Mendlovic, D., Zalevsky, Z., Garcia, J. \& Garcia Martinez, P. Superresolving Optical System with Time Multiplexing and Computer Decoding. Appl. Opt. 38, 7245-7251 (1999).

30. Kerschensteiner, M., Schwab, M. E., Lichtman, J. W. \& Misgeld, T. In vivo imaging of axonal degeneration and regeneration in the injured spinal cord. Nature Medicine 11, 572-577 (2005).

31. Vanzetta, I. \& Grinvald, A. Increased Cortical Oxidative Metabolism Due to Sensory Stimulation: Implications for Functional Brain Imaging. Science 286, 1555-1558 (1999).

32. Haidarliu, S., Sosnik, R. \& Ahissar, E. "Simultaneous multi-site recordings and iontophoretic drug and dye applications along the trigeminal system of anesthetized rats". J. of Neuroscience Methods 94 (1), 27-40 (1999).

33. Dunn, A. K. et al. Simultaneous imaging of total cerebral hemoglobin concentration, oxygenation, and blood flow during functional activation. Opt. Lett. 28, 28-30 (2003).

\section{Acknowledgments}

The authors would like to acknowledge the financial support given to this research from NIH grant R21 5R21EB009138-02 entitled "Novel nano-pipette for imaging of deep cortical layers and deep brain structures". Zeev Zalevsky also gratefully acknowledges the Leon and Maria Taubenblatt Prize for Excellence in Medical Research for supporting his research activity.

\section{Author contributions}

A.S. designed the device and the numerical testing, built the experimental setup and performed the experimental work and wrote the algorithm for computing the hemoglobin concentrations. S.A. and O.K. prepared the rats for the experiments. M.H. prepared the phantom. H.S. designed the rat experiments. A.D. designed the phantom experiments. Z.Z. initiated the project, designed the device and the experiments and supervised the entire project. The manuscript was written by A.S., A.D. and Z.Z.

\section{Additional information}

Supplementary information accompanies this paper at http://www.nature.com/ scientificreports

Competing financial interests: The authors declare no competing financial interests.

License: This work is licensed under a Creative Commons

Attribution-NonCommercial-NoDerivs 3.0 Unported License. To view a copy of this license, visit http://creativecommons.org/licenses/by-nc-nd/3.0/

How to cite this article: Shahmoon, A. et al. In vivo minimally invasive interstitial multi-functional microendoscopy. Sci. Rep. 3, 1805; DOI:10.1038/srep01805 (2013). 